\title{
One-step bone marrow-derived cell transplantation in talarosteochondral lesions: mid-term results
}

\author{
ROBERTO BUDA, FRANCESCA VANNINI, MARCO CAVALLO, MATTEO BALDASSARRI, \\ SIMONE NATALI, FRANCESCO CASTAGNINI, SANDRO GIANNINI
}

$1^{\text {st }}$ Orthopaedics and Traumatology Clinic, Rizzoli Orthopaedics Institute, Bologna, Italy

\begin{abstract}
Purpose: to verify the capability of scaffold-supported bone marrow-derived cells to be used in the repair of osteochondral lesions of the talus.

Methods: using a device to concentrate bone marrowderived cells, a scaffold (collagen powder or hyaluronic acid membrane) for cell support and platelet gel, a one-step arthroscopic technique was developed for cartilage repair. In a prospective clinical study, we investigated the ability of this technique to repair talar osteochondral lesions in 64 patients. The mean follow-up was 53 months. Clinical results were evaluated using the American Orthopaedic Foot and Ankle Society (AOFAS) scale score. We also considered the influence of scaffold type, lesion area, previous surgery, and lesion depth.

Results: the mean preoperative AOFAS scale score was $65.2 \pm 13.9$. The clinical results peaked at 24 months, before declining gradually to settle at a score of around 80 at the maximum follow-up of 72 months.

Conclusions: the use of bone marrow-derived cells supported by scaffolds to repair osteochondral lesions of the talus resulted in significant clinical improvement, which was maintained over time.

Level of Evidence: level IV, therapeutic case series.
\end{abstract}

\footnotetext{
Corresponding Author:

Francesca Vannini MD, PhD

$1^{\text {st }}$ Orthopaedics and Traumatology Clinic,

Rizzoli Orthopaedics Institute

Via di Barbiano 1/10, 40136 Bologna, Italy

Phone: +39 051/6366669

E-mail: France_vannini@yahoo.it
}

Key Words: bone marrow-derived cells, cartilage, osteochondral defect, repair, talus.

\section{Introduction}

Osteochondral lesions of the talus frequently occur after inversion or eversion ankle sprains in young patients participating in sports activities $(1,2)$. Since chondral tissue has poor healing abilities (3), the damage may be irreversible (4) and lead to chronic symptoms and early osteoarthritis, with a reported frequency ranging from 17 to $50 \%(5-7)$.

Various surgical options have been proposed to restore an adequate cartilaginous layer on the talar dome (8), but of these, only autologous chondrocyte implantation and osteochondral grafting have shown the ability to repair the lesion site with hyaline cartilage (9).

Tissue-engineering approaches have been suggested, including the use of biodegradable scaffolding, such as Hyalograft C (Fidia Advanced Biopolymers, Abano Terme, Italy) for cell support and proliferation, and matrix-induced autologous chondrocyte implantation. These procedures allow entirely arthroscopic autologous chondrocyte implantation with a substantial reduction in surgical morbidity (10-14). However, autologous chondrocyte implantation continues to have drawbacks, namely the need for two operations and the high costs involved $(12,13)$. The major disadvantages of osteochondral grafting, on the other hand, are the limited donor area available, the risk of damage to a healthy joint during the plug harvesting phase, possible discontinuous cartilage repair at the recipient site owing to slight discrepancies in plug orientation, and the need for open surgery at the 
donor and recipient sites, a malleolar osteotomy usually being required $(15,16)$. These considerations have prompted the search for new cartilage repair methods able to overcome these disadvantages. Recently, mesenchymal stem cells (MSCs), because of their ability to differentiate into various lineages, including osteoblasts and chondrocytes $(17,18)$, were proposed as a new option for the treatment of articular cartilage defects (19). MSC differentiation in the desired direction may be achieved as a result of environment, mechanical stimulation, and growth factors present in the platelet gel, capable of stimulating cells toward osteogenesis and chondrogenesis $(13,20,21)$. Although the use of MSCs as a pure cell lineage has been advocated (19), the key role in the described technique, following the selective elimination of cells that do not express the typical markers of MSCs, is played by the surrounding microenvironment (or niche).

The potential of a multipotent cell may be considered not only in terms of its intrinsic capability, but also in terms of its interaction with its physiological niche, which provides a signaling network (i.e., the extracellular matrix, adhesion molecules, growth factors, cytokines, and chemokines secreted by the resident cells) (22-24). Autologous bone marrow contains not only stem cells and precursor cells as a source of regenerative tissue, but also accessory cells that support angiogenesis and vasculogenesis by producing several growth factors. This suggests that, contrary to the case of autologous chondrocyte implantation, there may be no need for cell selection and expansion in the laboratory, and consequently that it may be possible to perform the transplant in a single surgical procedure.

Using a kit to concentrate bone marrow-derived cells in the operating room, collagen powder or hyaluronic acid membrane (as scaffolds for cell support) and platelet gel, a one-step arthroscopic technique was developed $(25,26)$.

The purpose of the present study was to verify whether the one-step arthroscopic technique using bone marrow-derived cells supported by different scaffolds and platelet gel to treat osteochondral lesions of the talus would improve the clinical, imaging and histological outcome.

The hypothesis of the study was that treatment of osteochondral lesions of the talus with bone marrowderived cells supported onto scaffolds may provide significant clinical improvement thanks the formation of repair tissue.

\section{Methods}

From 2006 to 2008, we enrolled 64 patients with focal osteochondral lesions of the talar dome for treatment with arthroscopic bone marrow-derived cell transplantation using a one-step technique and one of two scaffolds. The treatment was indicated for focal osteochondral lesions of the talar dome classified as chronic type II ( $1.5 \mathrm{~cm}^{2}$ in area, $<5 \mathrm{~mm}$ deep) (27). We excluded patients younger than 15 years or older than 50 years, patients with osteoarthritis or kissing lesions of the ankle, and patients with rheumatoid arthritis. Malalignment of the lower limb and the presence of joint laxity were considered relative contraindications to be corrected if present. The ethics committee of our institution approved the human protocol for this investigation.

The patient sample comprised 34 men and 30 women with a mean age of $30.5 \pm 10.5$ years. All the patients were affected by post-traumatic type II lesions (27).

The initial clinical evaluation of each patient included a complete history and a physical examination. We recorded any known cause of ankle trauma and previous failed treatment attempts. Two researchers involved in the study checked the ankle for instability, malalignment, and range of movement and calculated the American Orthopaedic Foot \& Ankle Society (AOFAS) scale score (28) preoperatively. A standard radiographic examination, including $\mathrm{AP}$ and lateral weight-bearing views, and MRI of the affected ankle were performed preoperatively.

The mean size of the lesions was $5.27 \pm 0.68 \mathrm{~cm}^{2}$, and their mean depth was $4.1 \pm 1.1 \mathrm{~mm}$. The lesion involved the right ankle in 31 patients and the left ankle in 33; the lesion was located medially in 49 and laterally in 15 . In 50 patients, the lesion had a definite posttraumatic origin. Six patients had a previous ankle fracture and 44 had a previous ankle sprain without fracture (mean time from the trauma, $37.4 \pm 48.5$ months), whereas 14 were not able to recall a specific 
major traumatic event, only various minor traumas to the affected ankle. Seventeen patients had been treated previously with: microfractures (eight patients), arthroscopic ankle debridement (six patients) and autologous chondrocyte implantation (three patients). Three patients presenting with a history of failed autologous chondrocyte implantation had previously undergone microfracture treatment (one) or arthroscopic debridement (two); therefore, the bone marrow-derived cell transplantation was these patients' third operation.

All investigations were conducted in conformity with ethical principles of research, and a written informed consent form was signed by all the patients enrolled in this study.

The scaffolds used for cell support in this series were either a porcine collagen powder (Spongostan 1 Powder; Johnson \& Johnson Medical Ltd, Gargrave, Skipton, UK), which, once mixed with autologous cell concentrate and platelet gel becomes a malleable paste, or a hyaluronic acid membrane (HYAFF1-11; Fidia Advanced Biopolymers) with the addition of platelet gel. According to commercial availability, the first 23 patients were treated using the collagen powder, whereas the following 41 patients received the hyaluronic acid membrane.

Before the clinical trial we performed a laboratory characterization of the implanted biomaterials to verify their in vitro capability to support bone marrow-derived cells. This was evaluated considering the viability and chondrogenic and osteogenic potential of the cells. The platelet-rich fibrin gel was produced the day before surgery using the Vivostat System1 (Vivolution A/S, 3460 Birkeroed, Denmark). We harvested and processed $120 \mathrm{ml}$ of the patient's venous blood to obtain 6 $\mathrm{ml}$ of platelet-rich fibrin gel. This product was cryopreserved at $-80{ }^{\circ} \mathrm{C}$ and returned to ambient temperature 30 minutes before the surgical procedure.

Bone marrow harvesting was performed in the same surgical session as the bone marrow-derived cell transplantation. The bone marrow was harvested from the posterior iliac crest in a sterile regimen, with the patient in the prone decubitus position and under general or spinal anesthesia. After insertion of a marrow needle (size, $11 \mathrm{G} 9100 \mathrm{~mm}$ ) $3 \mathrm{~cm}$ deep into the spongy bone, only $5 \mathrm{ml}$ of bone marrow was aspirated into a 20 -ml plastic syringe internally coated with cal- cium-heparin solution. The needle was then rotated 90 degrees and another $5 \mathrm{ml}$ was harvested. Additional harvestings of $5 \mathrm{ml}$ were obtained by rotating and slightly withdrawing the needle in the same location. This procedure was repeated with three to four perforations into the iliac crest, through the same skin opening, until a total of $60 \mathrm{ml}$ of bone marrow aspirate had been collected. The marrow was aspirated in small fractions from different points to maximize harvesting of marrow stromal cells and reduce dilution by peripheral blood. The harvested bone marrow was reduced in volume directly in the operating room by removing most of the red cells and plasma. In this way, it was possible to obtain a concentrate of nucleated cells, including stem cells, monocytes, lymphocytes, and other bone marrow resident cells. The bone marrow was concentrated with a cell separator-concentrator (Smart PReP1; Harvest Technologies Corp, Plymouth, MA) using a sterile and disposable dedicated kit (BMAC1; Harvest Technologies Corp). The $60 \mathrm{ml}$ of bone marrow aspirate to be processed (the minimum amount required by the manufacturer) was injected into the posterior portion of the double chamber device. The device was placed vertically on the rotor of the centrifuge. A three-minute centrifugation at $2500 \mathrm{rpm}$ separated the red cells from the other components with the help of a semipermeable membrane; then, during a two-minute arrest period, the buffy coat separated from the erythrocytes was transferred into the anterior chamber. Finally, a nine-minute phase at $2300 \mathrm{rpm}$ separated the cellular component from the plasma in the anterior chamber; at the end of the entire process, the majority of the red cells remained in the posterior chamber of the device. Once separated from the red cells, the mononuclear cells remained at the bottom of the anterior chamber covered by the platelet-poor plasma. Finally, they were aspirated from the anterior chamber with a syringe and re-suspended in an adequate volume of the platelet-poor plasma to obtain $6 \mathrm{ml}$ of cell concentrate.

After the bone marrow harvesting phase, we performed ankle arthroscopy with the patient in a supine position with a tourniquet at $280-\mathrm{mm} \mathrm{Hg}$ pressure applied at the thigh of the affected limb. Two standard approaches, anteromedial and anterolateral, were used. The lesion site was observed and prepared by removing the 
detached cartilage fragment and subchondral malacic bone until the healthy bone was reached. With the help of a calibrated probe, we measured the lesion size. Then, the composites to be implanted were prepared. When collagen was used, $1 \mathrm{~g}$ powder was mixed with 2 $\mathrm{ml}$ bone marrow concentrate and $1 \mathrm{ml}$ platelet-rich fibrin gel to create a malleable paste rich in bone marrow progenitor cells and growth factors. Instead, the hyaluronic acid membrane was cut to an appropriate size following the area of the lesion and loaded by capillary action with $2 \mathrm{ml}$ bone marrow concentrate and 1 $\mathrm{ml}$ platelet-rich fibrin gel. The composites were placed using the same instrumentation described elsewhere for arthroscopic autologous chondrocyte implantation (CITIEFFE Srl, Calderara di Reno, Italy) (11) (Fig. 1). The cannula was inserted through the arthroscopic portal closest to the lesion with the help of the dedicated trochar, the distension liquid flow was stopped, and the liquid was removed from the articular environment. The final composite was run through the cannula onto a sliding positioner up to the margin of the lesion, where it was positioned with the help of a probe. We then removed the cannula and carefully fitted the composite into the lesion site using a flattened probe (Fig. 2). A layer of platelet-rich fibrin gel then was spread on the implant to provide an additional supply of growth factors and to optimize its stability

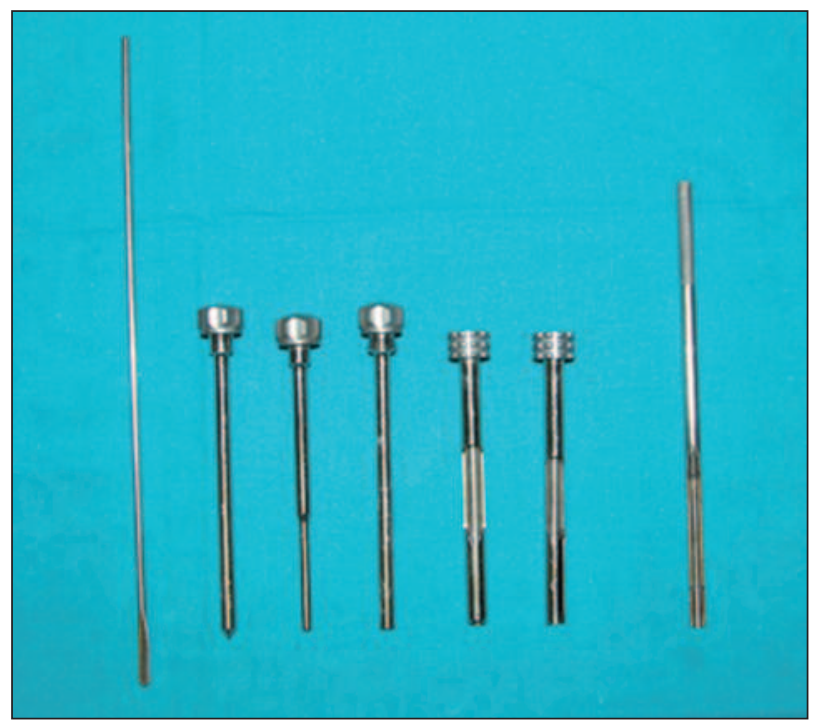

Fig. 1. Surgical instruments originally designed for autologous chondrocyte implantation and then adapted for bone marrow-derived cell transplantation.

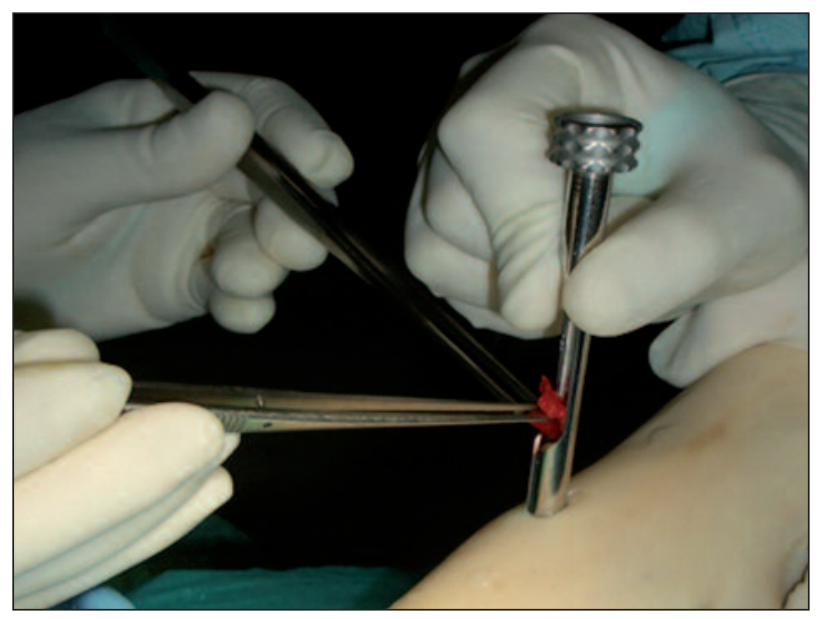

Fig. 2. Implantation of the biomaterials using the cannula.

through the clotting capability of the gel. We performed multiple sagittal ankle movements under arthroscopic control to verify the stability of the implant. Twenty-two patients underwent associated surgical procedures: osteophytectomy in 17 , synovectomy for synovial hypertrophy in two, loose body extraction in two, and calcaneal osteotomy for flatfoot correction in one. The skin was sutured using absorbable 3-0 suture, with a single stitch for each portal.

Active and passive ankle motions were recommended the day after surgery. The range of motion was increased gradually according to pain tolerance for the first three weeks. The patients were advised to walk with crutches and to avoid weight bearing on the affected ankle for the first six weeks after surgery.

Partial weight bearing increasing to complete weight bearing was permitted from six to eight weeks after surgery, while low-impact sports activity could be resumed four months after surgery. At 10 to 12 months, we allowed running and progressive training for high-impact activities such as tennis and soccer.

Patients had clinical follow-ups at 6,12, 18, and 24 months after surgery, and every year thereafter, until the maximum follow-up of 72 months. Clinical outcome at the follow-up visits was assessed on the basis of the AOFAS score and the patient's return to sports activities. Results were compared with baseline values and analyzed according to the following predictors: type of scaffold, lesion area and depth, previous surgical procedures at the same lesion site. 


\section{Results}

The mean follow-up duration was 53 months. No patients were lost to follow-up.

We encountered no intraoperative complications. Postoperatively, one patient had a superficial infection of an arthroscopic portal, which resolved with oral antibiotic therapy.

The mean preoperative AOFAS scale score was $65.2 \pm$ 13.9. The mean AOFAS score improved to $83.7 \pm 9.1$ at 6 months' follow-up, $89.1 \pm 8.1$ at 12 months, 89.2 \pm 9.5 at 18 months, and $91.1 \pm 8.7$ at 24 months. At 36 months, the mean AOFAS score was $85.6 \pm 10.1$, at 42 months $82.4 \pm 12$, at 60 months $80 \pm 13.5$, and finally at 72 months $80.7 \pm 14.1$. Sixty of the 64 patients (94\%) were participating in a low-impact sports activity at a mean of 4.8 months after the surgery, whereas 49 of the 64 patients $(77 \%)$ were participating in a high-impact sports activity at a mean of 11.9 months. All the patients showed a similar pattern of improvement at each follow-up, regardless of the scaffold type used (collagen powder in 21 patients and hyaluronic membrane in 43).

The percentages of maximum possible improvement also were similar between the groups. As the results were not affected by the type of scaffold used, the following correlations were calculated in the whole group of patients.

We observed no correlation between the area of the lesion and the preoperative AOFAS score, but did observe a relationship between the area of the lesion and the AOFAS score at each follow-up. We also observed a relationship between the area of the lesion and the percentage of maximum possible improvement in the AOFAS score at each follow-up. The percentage of maximum possible improvement in the AOFAS score was similar between the two groups at each follow-up. No relationship was observed between the AOFAS score and the depth of the lesion.

\section{Discussion}

Autologous chondrocyte implantation is widely considered the state of the art in cartilage regeneration, having shown its ability to regenerate reparative tissue with physical characteristics similar to those of healthy cartilage (18). Furthermore, the use of a tissue-engineered biomaterial composed of autologous chondrocytes grown on a scaffold made entirely of HYAFF111 has been found to enhance the applicability of the technique, permitting a completely arthroscopic procedure in the knee and ankle $(11,29)$. Nevertheless, the need for two surgical operations and the high costs of cell expansion are major drawbacks of this technique, which may never be overcome. Furthermore, chondrocytes are differentiated cells, which are able to regenerate only cartilaginous tissue.

The rationale of the present study is based on the hypothesis that bone marrow-derived cells may be used to repair osteochondral lesions of the talus, overcoming all the drawbacks associated with autologous chondrocyte implantation.

The clinical results, after peaking at 24 months, declined gradually to settle at an AOFAS score of around 80 at the maximum follow-up of 72 months. The main parameter influencing the final functional score was the area of the lesion. The presence of previous surgery negatively influenced the score preoperatively and postoperatively, whereas no differences in percentage of clinical improvement were found between patients who had and those who had not previously undergone surgery.

The use of two different bone marrow-derived cell scaffolds (due to the commercial availability of the materials during the study) is a weakness of this study. Nevertheless, the results obtained with the two scaffolds showed a similar pattern of improvement, indicating that they provide equivalent support for transplanted bone marrow-derived cells.

Transplantation of the entire bone marrow cellular pool, thereby transferring the entire regenerative potential present in the bone marrow to the lesion site, allows the cells to be processed directly in the operating room without the need for a laboratory phase and thus allows the technique to be performed in a single step. Although longer follow-up is needed to confirm the validity of the repair over time, this arthroscopic one-step technique represents an advance in osteochondral regeneration, achieving high clinical scores, without the major disadvantages of previous techniques. 


\section{References}

1. Bosien WR, Staples OS, Russell SW. Residual disability following acute ankle sprains. J Bone Joint Surg Am 1955; 37:1237-1243.

2. Pritsch M, Horoshovski H, Farine I. Arthroscopic treatment of osteochondral lesions of the talus. J Bone Joint Surg Am 1986;68:862-865.

3. Aigner J, Tegeler J, Hutzler P, et al. Cartilage tissue engineering with novel nonwoven structured biomaterial based on hyaluronic acid benzyl ester. J Biomed Mater Res 1998; 42:172-181.

4. Buckwalter JA, Lohmander S. Operative treatment of osteoarthrosis. Current practice and future development. J Bone Joint Surg Am 1994;76:1405-1418.

5. Bartlett W, Skinner JA, Gooding CR, et al. Autologous chondrocyte implantation versus matrix-induced autologous chondrocyte implantation for osteochondral defects of the knee: a prospective, randomized study. J Bone Joint Surg Br 2005;87:640-645.

6. Buckwalter JA, Mow VC, Ratcliffe A. Restoration of injured or degenerated articular cartilage. J Am Acad Orthop Surg 1994;2:192-201.

7. Canale ST, Belding RH. Osteochondral lesions of the talus. J Bone Joint Surg Am 1980;62:97-102.

8. Moran ME, Kim HK, Salter RB. Biological resurfacing of full-thickness defects in patellar articular cartilage of the rabbit: investigation of autogenous periosteal grafts subjected to continuous passive motion. J Bone Joint Surg Br 1992;74: 659-667.

9. Brittberg M. Autologous chondrocyte transplantation. Clin Orthop Relat Res 1999;(367 Suppl):S147-S155.

10. Ferruzzi A, Buda R, Faldini C, et al. Autologous chondrocyte implantation in the knee joint: open compared with arthroscopic technique. Comparison at a minimum follow-up of five years. J Bone Joint Surg Am 2008;90(Suppl 4):90-101.

11. Giannini S, Buda R, Vannini F, et al. Arthroscopic autologous chondrocyte implantation in osteochondral lesions of the talus: surgical technique and results. Am J Sports Med 2008;36:873-880.

12. Giannini S, Buda R, Grigolo B, et al. Autologous chondrocyte transplantation in osteochondral lesions of the ankle joint. Foot Ankle Int 2001;22:513-517.

13. Giannini S, Buda R, Grigolo B, et al. The detached osteochondral fragment as a source of cells for autologous chondrocyte implantation (ACI) in the ankle joint. Osteoarthritis Cartilage 2005;13:601-607.

14. Giannini S, Vannini F. Operative treatment of osteochondral lesions of the talar dome: current concepts review. Foot Ankle Int 2004;25:168-175.
15. Hangody L, Feczkó P, Bartha L, et al. Mosaicplasty for the treatment of articular defects of the knee and ankle. Clin Orthop Relat Res 2001;(391 Suppl):S328-S336.

16. Kreuz PC, Steinwachs M, Erggelet C, et al. Mosaicplasty with autogenous talar autograft for osteochondral lesions of the talus after failed primary arthroscopic management: a prospective study with a 4-year follow-up. Am J Sports Med 2006;34:55-63.

17. Galois L, Freyria AM, Herbage D, et al. Cartilage tissue engineering: state-of-the-art and future approaches. Pathol Biol (Paris) 2005;53:590-598

18. Oreffo RO, Cooper C, Mason C, et al. Mesenchymal stem cells: lineage, plasticity, and skeletal therapeutic potential. Stem Cell Rev 2005;1:169-178.

19. Beris AE, Lykissas MG, Papageorgiou CD, et al. Advances in articular cartilage repair. Injury 2005;36(Suppl 4):S14-S23.

20. Schutze N, Noth U, Schneidereit J, et al. Differential expression of CCN-family members in primary human bone marrow-derived mesenchymal stem cells during osteogenic, chondrogenic and adipogenic differentiation. Cell Commun Signal 2005;3:5.

21. White LM, Sussman MS, Hurtig M, et al. Cartilage T2 assessment: differentiation of normal hyaline cartilage and reparative tissue after arthroscopic cartilage repair in equine subjects. Radiology 2006;241:407-414.

22. Dominici M, Pritchard C, Garlits JE, et al. Hematopoietic cells and osteoblasts are derived from a common marrow progenitor after bone marrow transplantation. Proc Natl Acad Sci U S A 2004;101:11761-11766.

23. Kacena MA, Gundberg CM, Horowitz MC. A reciprocal regulatory interaction between megacaryocytes, bone cells and hematopoietic stem cells. Bone 2006;39:978-984.

24. Olmsted-Davis EA, Gugala Z, Camargo F, et al. Primitive adult hematopoietic stem cells can function as osteoblast precursors. Proc Natl Acad Sci U S A 2003;100: 15877-15882.

25. Caplan AI. Adult mesenchymal stem cells for tissue engineering versus regenerative medicine. J Cell Physiol 2007;213: 341-347.

26. Lucarelli E, Beccheroni A, Donati D, et al. Platelet-derived growth factors enhance proliferation of human stromal stem cells. Biomaterials 2003;24:3095-3100.

27. Giannini S, Buda R, Faldini C, et al. Surgical treatment of osteochondral lesions of the talus in young active patients. J Bone Joint Surg Am 2005;87(Suppl 2):28-41.

28. Kitaoka HB, Alexander IJ, Adelaar RS, et al. Clinical rating systems for the ankle-hindfoot, midfoot, hallux, and lesser toes. Foot Ankle Int 1994;15:349-353.

29. Marcacci M, Berruto M, Brocchetta D, et al. Articular cartilage engineering with Hyalograft C: 3-year clinical results. Clin Orthop Relat Res 2005;(435):96-105. 